\title{
Dual-phase argon ionization detector for measurement of coherent elastic neutrino scattering and medium-energy nuclear recoils
}

\author{
Winant, C.D. ${ }^{(1,2)}$, Bernstein, A. ${ }^{(1)}$, Hagmann, C. ${ }^{(1)}$, Madden, N. $^{(1)}$, Stoeffl, W. ${ }^{(1)}$ \\ (1) Lawrence Livermore National Laboratory, 7000 East Ave., Livermore CA 94550 \\ (2)winant2@1lnl.gov \\ UCRL-PROC-224459
}

\begin{abstract}
We propose to build and deploy a 10-kg dual-phase argon ionization detector for the detection of coherent neutrino-nucleus scattering, which is described by the reaction; $(v)+(\mathrm{Z}, \mathrm{N}) \rightarrow(v)+(\mathrm{Z}, \mathrm{N})$. Our group would be the first to make this measurement. Its detection would validate (or refute) central tenets of the Standard Model. The existence of this process is also relevant to astrophysics, where coherent neutrino scattering is assumed to impede energy transport within neutron stars. We have built a gas-phase argon ionization detector to determine the feasibility of measuring small recoil energies $(\sim 1 \mathrm{keV})$ predicted from coherent neutrino scattering, and to characterize the recoil spectrum of the argon nuclei induced by scattering from medium-energy neutrons. We present calibrations made with $55-\mathrm{Fe}$, a low energy x-ray source, and describe a planned measurement of the recoil spectra from the $60 \mathrm{keV}$ Lithium-target neutron generator at LLNL. A high signal-tonoise measurement of the recoil spectrum will not only serve an important milestone in achieving the sensitivity necessary for measuring coherent neutrino-nucleus scattering, but will break new scientific ground by providing a first ever measurement of low-energy quenching factors in argon.
\end{abstract}

Coherent scattering occurs when the momentum transfer from a neutrino to the nucleus is much smaller than the inverse size of the recoil nucleus. A detection of coherent neutrino-nucleus scattering would verify an unconfirmed Standard Model prediction [1], explore non-standard neutrino-quark interactions, confirm stellar collapse and supernova energy transport and neutrino opacity models, and could be applied to the measurement of the flavor-blind neutrino spectrum from next nearby supernova, or could be used to promote non-intrusive reactor power monitoring [2].

We propose detecting the ionization induced by recoiling argon nuclei using a $10 \mathrm{~kg}$ dualphase argon detector. The principle of dual-phase detection has been described elsewhere [3]. We propose using a $3 \mathrm{GW}$ commercial nuclear reactor as a source of antineutrinos. We have designed and built a gas-phase prototype of the detector with which we have measured the 200 -electron equivalent ionization signals from a $6 \mathrm{keV} \mathrm{Fe}-55$ source with a signal-to-noise threshold of 50 electrons. This prototype also enables study of scintillation properties of Argon and investigation of electron and nuclear recoils in Argon. We will measure medium energy neutron-nuclear recoils in our prototype detector using the recently-commissioned LLNL compact pulsed neutron source.

\section{References}

[1] Freedman, D. Phys. Rev. D 9, 1389-1392 (1974)

[2] Hagmann, C. A., Bernstein, A. IEEE transactions on Nuclear Science, 51(5), 2151 (2004)

[3] Bolozdynya, A.I., NIM A 422, 314-320 (1999)

This work was performed under the auspices of the U. S. Department of Energy by University of California, Lawrence Livermore National Laboratory under contract W-7405-Eng-48. 